\title{
Editorial
}

Nephrology

\section{Tobacco Use: A Chronic Kidney Disease Accelerant}

\author{
Amanda K. Leonberg-Yoo Michael R. Rudnick \\ Division of Nephrology, Perelman School of Medicine at the University of Pennsylvania, Philadelphia, PA, USA
}

Cigarette smoking is a modifiable risk factor for several chronic disease states, including cardiovascular disease, cancer, and pulmonary diseases. The association between cigarette smoking and chronic kidney disease (CKD) has also been evaluated in observational settings, with most studies showing a positive relationship between smoking and CKD $[1,2]$.

The adverse effects of smoking on kidney function have been demonstrated among a general population and CKD-specific population. In the Cardiovascular Health Study cohort, smoking was independently associated with an increase in serum creatinine (OR 1.31 [1.12-1.52] for every 5 cigarettes per day, resulting in a $0.3 \mathrm{mg} / \mathrm{dL}$ rise in serum creatinine) [3]. In patients with diabetes mellitus, IgA nephropathy, and renal transplants, smoking is also associated with an accelerated reduction in glomerular filtration rate (GFR) [1]. In the Diabetes Control and Complications trial, the rate of GFR decline in patients with type 1 diabetes was 4.3 times greater in smokers compared to nonsmokers [4]. Prior studies have also shown that there may be a dose-dependent response, with increased smoking exposure leading to a stronger association. A cross-sectional study in 65,193 individuals demonstrated that an increased CKD risk in those with a cumulative lifetime cigarette exposure of either 25-49 or $>50$ pack-years was RR 1.42 and 2.05, respectively [5]. Only a few studies have been performed to identify the impact of smoking cessation on renal disease progression in a CKD population $[1,6]$.

Wesson et al. conducted a prospective study in 216 hypertensive patients with CKD stage 2 with albuminuria (albumin:creatinine ratio $>200 \mathrm{mg} / \mathrm{g}$ ) to test the hypothesis whether smoking cessation restores ACE inhibition (ACEI), kidney protection against progression compared to those who continued smoking and nonsmokers [7]. Individuals with diabetes, systolic blood pressure (BP) $>200 \mathrm{~mm} \mathrm{Hg}$, history of malignancy or transplant, or inability to tolerate ACEIs were excluded. Individuals who had limited smoking exposure $(<10$ cigarettes per day or who had discontinued smoking $<1$ year ago, or nonsmokers who last smoked 1 year before recruitment) were also excluded. Current smokers were defined as those consuming $\geq 10$ cigarettes per day for $\geq 1$ year. All smokers were offered pharmacologic and non-pharmacologic smoking cessation interventions. Smoking cessation was defined as a reduction of urine and plasma cotinine at 24 weeks to $<10 \%$, compared to baseline. Of the 108 individuals enrolled who smoked, $23 \%(n=25)$ ceased smok-

\section{KARGER}

(c) 2017 S. Karger AG, Basel

E-Mail karger@karger.com

www.karger.com/ajn
Amanda Leonberg-Yoo, MD

Penn Presbyterian Medical Center, Division of Nephrology

Medical Office Building Suite 240, 51 N. 39th Street

Philadelphia, PA 19104 (USA)

E-Mail Amanda.Leonberg-Yoo@uphs.upenn.edu 
ing and were defined as quitters. Study participants were followed prospectively for 5 years, with yearly blood work, urine studies, and BP measurements.

Recruited patients were started on enalapril ( $97 \%$ not on ACEI at baseline), with dose titration for a targeted BP of $<130 / 80 \mathrm{~mm} \mathrm{Hg}$ (average dose 12-14 mg/day). Individuals who enrolled in the study were predominantly of black ethnicity, on 2 or more BP lowering drugs, with similar baseline estimated GFR (eGFR) around $74 \mathrm{~mL} /$ $\min / 1.73 \mathrm{~m}^{2}$ ( $p=0.92$ between groups) and similar baseline urine albumin $(p=0.30)$. The cumulative cigarette exposure was lower in smokers who quit, as compared to those who continued smoking, which was measured by the Brinkman Index (cigarettes per day $\times$ number of years smoked). There was no difference in systolic and diastolic BP between each group at baseline or year 5, and all groups observed the intended reduction in BP.

The primary outcome was the change in eGFR over time, estimated by cystatin-based CKD-EPI equation. Secondary outcomes included the change in albumin excretion as a marker of kidney damage and urine isoprostane 8-isoprostaglandin F2a (U8-iso) and urine angiotensinogen (UATG) as markers of oxidative stress and angiotensin II kidney activity, respectively. All 3 groups demonstrated a significant decline in eGFR over time. Among nonsmokers, the average yearly decline in eGFR was lowest $\left(1.3 \pm 1.5 \mathrm{~mL} / \mathrm{min} / 1.73 \mathrm{~m}^{2} /\right.$ year $)$ as compared to quitters $\left(1.7 \pm 1.5 \mathrm{~mL} / \mathrm{min} / 1.73 \mathrm{~m}^{2} /\right.$ year $)$ but this difference was not significant $(p=0.06)$. The largest decline in eGFR was observed in current smokers, with a reduction in eGFR of $3.4 \pm 1.8 \mathrm{~mL} / \mathrm{min} / 1.73 \mathrm{~m}^{2} /$ year $(p<0.0001$ compared to nonsmokers). At year one, albuminuria was reduced in nonsmokers; however, there was no difference in those that quit smoking, and there was an increase in albuminuria among continued smokers. Five-year albuminuria was higher than baseline for all groups; however, those that continued smoking had significantly higher levels, as compared to those that were nonsmokers and quitters.

The major findings of the study include the observation that continued smoking is associated with accelerated GFR decline. Current smokers had the lowest eGFR at year 5 , as compared to quitters and nonsmokers. Smoking cessation attenuated this eGFR reduction as compared to current smokers $(-1.7 \pm 1.5$ vs. $-3.4 \pm 1.8 \mathrm{~mL} /$ $\mathrm{min} / 1.73 \mathrm{~m}^{2} /$ year $)$, with a similar rate of change in eGFR per year as compared to nonsmokers $(-1.3 \pm 1.5 \mathrm{~mL} /$ $\mathrm{min} / 1.73 \mathrm{~m}^{2} /$ year). Albuminuria increased over time in all groups, with the greatest rate of increase in continued smokers. Interestingly, the rate of change of albuminuria in those that quit smoking mimicked the rate of change in nonsmokers, with both groups observing a lesser rise in albuminuria over the 5-year study period.

The authors propose a causal mechanism of cigarette smoking and CKD progression via increased oxidative stress and activation of the renin-angiotensin system despite ACEI. At 5 years, UATG increased in all groups, with continued smokers having the greatest increase $(p<$ 0.001 ) despite similar baseline levels in all groups. This increased UATG was sustained, despite the addition of ACEI, suggesting that conversion to angiotensin II may occur independent of the angiotensin converting enzyme inhibition, or that the dose of ACEI was not adequate. Oxidative stress as a mechanism of smoking injury to the kidney was supported by the observation that U8-iso levels were highest at baseline in smokers and quitters and lowest in nonsmokers. At follow up, U8-iso levels remained high in smokers but fell in quitters to a level similar to nonsmokers. Other mechanisms, including renal hemodynamic and peripheral vascular changes, were not studied and may also play a role in the observed effect of smoking and GFR decline.

The greatest strength of the study is the inclusion of a cohort of smokers who discontinued tobacco. This group allowed the authors to evaluate the effect of smoking cessation, of which, little has been published. Second, the authors developed a cohort of patients enriched for smoking exposure with a high likelihood of CKD progression, given the presence of albuminuria, with appropriate follow-up time of 5 years to determine the difference in measured outcomes. Third, the primary outcome, change in eGFR, was chosen as a clinically meaningful outcome, given the short-term follow-up and low likelihood of progression to clinical outcomes, including death or endstage renal disease. The use of cystatin-based estimating equations prevents the misclassification bias from creatinine-based equations, which have been shown to overestimate the renal function in smokers [6].

The study also has some weaknesses. The authors did not account for a dose-dependent relationship between smoking and CKD progression or albuminuria. Those that quit smoking had less cumulative exposure at baseline, as compared to continued smokers, thus potentially accounting for the more favorable outcomes in eGFR and albuminuria in quitters, independent of the smoking cessation intervention. Second, the study was an observational prospective study that monitored 3 smoking exposure groups over time, but did not adjust for known confounders of CKD progression. Although descriptive in nature, the study could have been strengthened by the 
evaluation of GFR decline, after adjustment for known confounders. Ostensibly, a strength of the study was to include the smoking cessation group; however, the number of individuals within this group was small $(n=25)$, potentially creating an imbalance of unmeasured confounders between the study groups that could have accounted for the differences in results. Finally, although the exclusion criteria removed nonsmokers with a prior smoking history of up to 1 year, there may be some dilution in the treatment effect of the nonsmokers because there may be some former smokers within this group, which may bias the results towards the null hypothesis of no difference between the nonsmoker group and the quitter group.

In summary, this study provides additional evidence in a hypertensive cohort at risk for CKD progression that continued smoking adversely affects kidney disease progression, as compared to those that did not smoke. Since the majority of prior studies of smoking and CKD are observational, the finding that smoking cessation resulted in a lesser decline in kidney function, as compared to active smokers in a prospective study design may be the most important contribution of this study. In addition, smoking cessation only partially restores the ACEI protection against CKD progression, as compared to nonsmokers, although additional studies using a larger cohort of quitters may be necessary for generating further conclusions. Public health efforts should continue to focus on the prevention of smoking for general health, as well as kidney health, and nephrologists should urge smoking cessation as a means to prevent $\mathrm{CKD}$ progression.

\section{Disclosure Statement}

The authors have no conflicts of interest.

\section{References}

1

Orth SR, Hallan SI: Smoking: a risk factor for progression of chronic kidney disease and for cardiovascular morbidity and mortality in renal patients - absence of evidence or evidence of absence? Clin J Am Soc Nephrol 2008;3:226-236.

2 Nagasawa $Y$, Yamamoto R, Rakugi H, Isaka Y: Cigarette smoking and chronic kidney diseases. Hypertens Res 2012;35:261-265.

3 Bleyer AJ, Shemanski LR, Burke GL, Hansen KJ, Appel RG: Tobacco, hypertension, and vascular disease: risk factors for renal functional decline in an older population. Kidney Intl 2000;57:2072-2079.
4 de Boer IH, Sibley SD, Kestenbaum B, et al: Central obesity, incident microalbuminuria, and change in creatinine clearance in the epidemiology of diabetes interventions and complications study. J Am Soc Nephrol 2007;18: 235-243.

5 Hallan S, de Mutsert R, Carlsen S, Dekker FW, Aasarød K, Holmen J: Obesity, smoking, and physical inactivity as risk factors for CKD: are men more vulnerable? Am J Kidney Dis 2006; 47:396-405.
6 Ohkuma T, Nakamura U, Iwase M, et al: Effects of smoking and its cessation on creatinine- and cystatin C-based estimated glomerular filtration rates and albuminuria in male patients with type 2 diabetes mellitus: the $\mathrm{Fu}$ kuoka Diabetes Registry. Hypertens Res 2016; 39:744-751.

7 Roehm B, Simoni J, Prusynski J, Wesson D: Cigarette smoking attenuates kidney protection by angiotensin-converting enzyme inhibition in nondiabetic chronic kidney disease. Am J Nephrol 2017;46:260-267. 